\title{
Papillary adenocarcinoma of the rete testis with adjacent hyperplasia: a case report
}

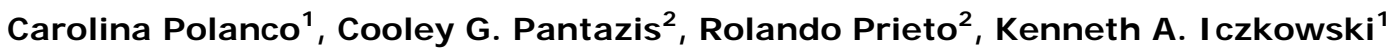 \\ 1. Medical College of Wisconsin, Milwaukee, WI, United States. 2. Munroe Regional Medical Center, Ocala, FL, United \\ States.
}

Correspondence: Kenneth A. Iczkowski. Address: Medical College of Wisconsin, United States. E-mail: kaiczkowski@mcw.edu

Received: October 14, 2014

Accepted: December 12, 2014

Online Published: December 18, 2014

DOI : $10.5430 / c r c p . v 2 n 2 p 41$

URL: http://dx.doi.org/10.5430/crcp.v2n2p41

\begin{abstract}
An 81-year-old man had a papillary adenocarcinoma infiltrating the rete testis with involvement of the epididymis and paratesticular soft tissue. The tumor was composed of papillary structures to focally solid areas with cells having abundant eosinophilic to clear cytoplasm, markedly atypical nuclei, and brisk mitotic activity. Invasive growth and extensive necrosis were present. Morphology, immunohistochemistry, and medical history support the diagnosis. Our case represents the second documented case with foci of adenomatous hyperplasia adjoining the tumor.
\end{abstract}

\section{Key words}

Rete testis, Adenocarcinoma, Metastatic carcinoma, Immunohistochemistry

\section{Introduction}

Primary carcinoma of the rete testis is extremely rare, with 30-60 cases reported in the literature. This entity occurs mostly in men older than 60 years. The clinical presentation is nonspecific; metastases are present at diagnosis in a large percent of patients. Some reported clinical manifestations include chronic epididymitis, hydrocele, and inguinal hernia ${ }^{[1]}$. Since the histologic diagnosis of this tumor is challenging, Nochomovitz and Orenstein have proposed histologic criteria, including: location of tumor in the mediastinum of the testis, demonstration that the neoplasm arises out of adjacent normal rete testis, that the tunica vaginalis is intact, that there is no other primary tumor, and a predominantly solid gross appearance $^{[2]}$.

\section{Clinical presentation}

An 81- year-old man presented with approximately five years' history of right swelling of the right scrotum. Past history was noncontributory, except for hypercholesterolemia. Past surgical history included neck surgery for unknown reason and hip replacement. Physical examination revealed a large nontender, right sided hemiscrotum. Ultrasonography of the scrotum (see Figure 1) revealed a $6.5 \mathrm{~cm}$ right spermatocele and a large right hydrocele. He underwent a right hydrocelectomy and spermatocelectomy. However, after those interventions, the scrotal swelling still persisted. Two months later the patient had a total orchiectomy and the specimen underwent diagnostic examination. 
Figure 1. Realtime sonography demonstrates a well-demarginated cystic septated lesion arising from the upper pole of the right testicle. The lesion measures $6.5 \mathrm{~cm} \times 5.6 \mathrm{~cm} \times 6.3 \mathrm{~cm}$ and contains a $1.9 \mathrm{~cm} \times 1.5 \mathrm{~cm} \times 1.8 \mathrm{~cm}$ mural basal nodule.

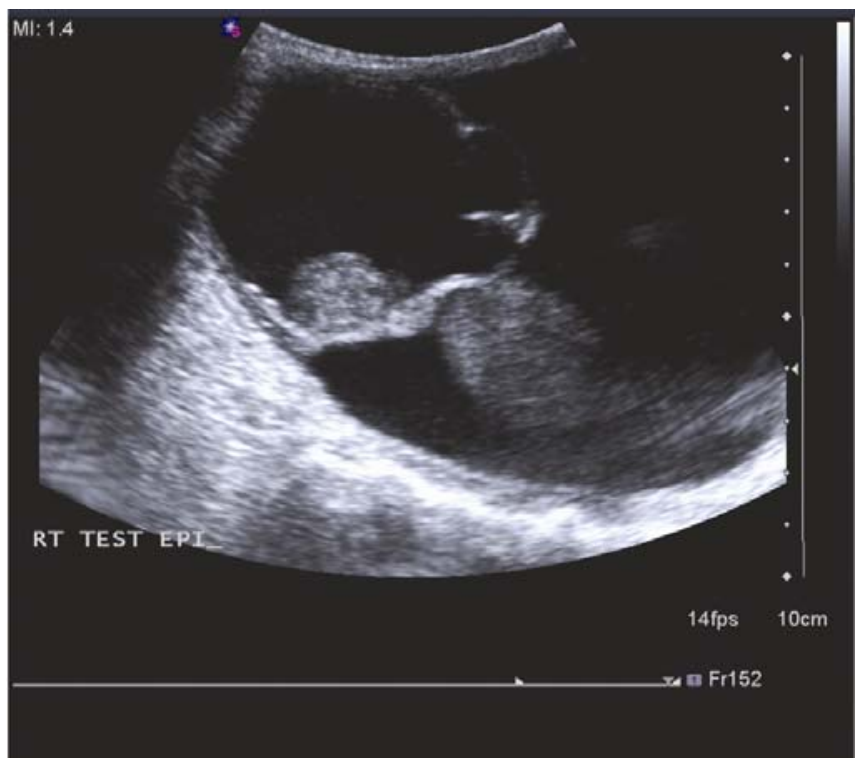

\section{Methods}

The resected specimen was formalin-fixed and paraffin-embedded. Besides standard slides, immunostained slides were prepared using these antibodies: CK8, AE1/AE3, Vimentin, WT-1, CK 7, OCT3/4, CD30, AFP, glypican 3, CD117, CD10, LeuM1, MOC-31, BerEP4, inhibin, CDX-2, TTF-1, p63, PSA, PLAP and ER.

\section{Results}

\subsection{Gross features}

The specimen was received in formalin and consisted of a left total orchiectomy with attached tunica vaginalis measuring $10 \mathrm{~cm} \times 8 \mathrm{~cm} \times 6 \mathrm{~cm}$ and weighing 174 grams. The tunica vaginalis revealed a $5.5 \mathrm{~cm} \times 5.0 \mathrm{~cm} \times 4.5 \mathrm{~cm}$ cystic mass, involving predominantly the head of the epididymis. Sectioning of the testicular parenchyma showed numerous red to yellow friable areas suggesting possible focal necrosis.

\subsection{Microscopic features}

Review of the standard sections revealed a tumor centered in and infiltrating around the rete tubules, composed partially of papillary structures lined by cuboidal to polygonal cells with abundant eosinophilic to clear cytoplasm, severe nuclear atypia, and numerous mitoses. The tumor showed a biphasic appearance; in some areas, the apical portions of the cells were vacuolated, imparting a pseudomicrocystic appearance. Numerous eosinophilic hyaline globules were also appreciated. In other areas of the tumor, the neoplastic cells formed solid sheets or long cribriform structures with abundant granular eosinophilic cytoplasm. Invasive growth and extensive necrosis were present (see Figure 2). Adenomatous hyperplasia was noted in adjacent foci (see Figure 3).

\subsection{I mmunohistochemistry}

Numerous immunostains performed at 2 different institutions showed tumor cells to react intensely for pancytokeratin, CK8, and vimentin, and they were focally positive for CD10 and LeuM1 (see Figure 4). Mesothelioma markers such as MOC-31 and BerEP4 demonstrated questionable weak staining. WT-1, CK7, and numerous germ cell markers including, OCT3/4, CD30, alpha-fetoprotein (AFP), PLAP, Glypican 3, and CD117 were negative. A calretinin immunostain performed in one institution was negative; however, the same immunostain was positive in the neoplastic cells at a second 
institution. Since the differential diagnosis for this tumor includes primary carcinoma of the rete testis, mesothelioma, a metastasis, mullerian type carcinoma of the testis, and a germ cell tumor, additional immunostains were performed, including inhibin $\alpha$, CDX-2, TTF-1, p63, prostate specific antigen (PSA), prostatic acid phosphatase (PLAP), and estrogen receptor (ER). All of these were negative.
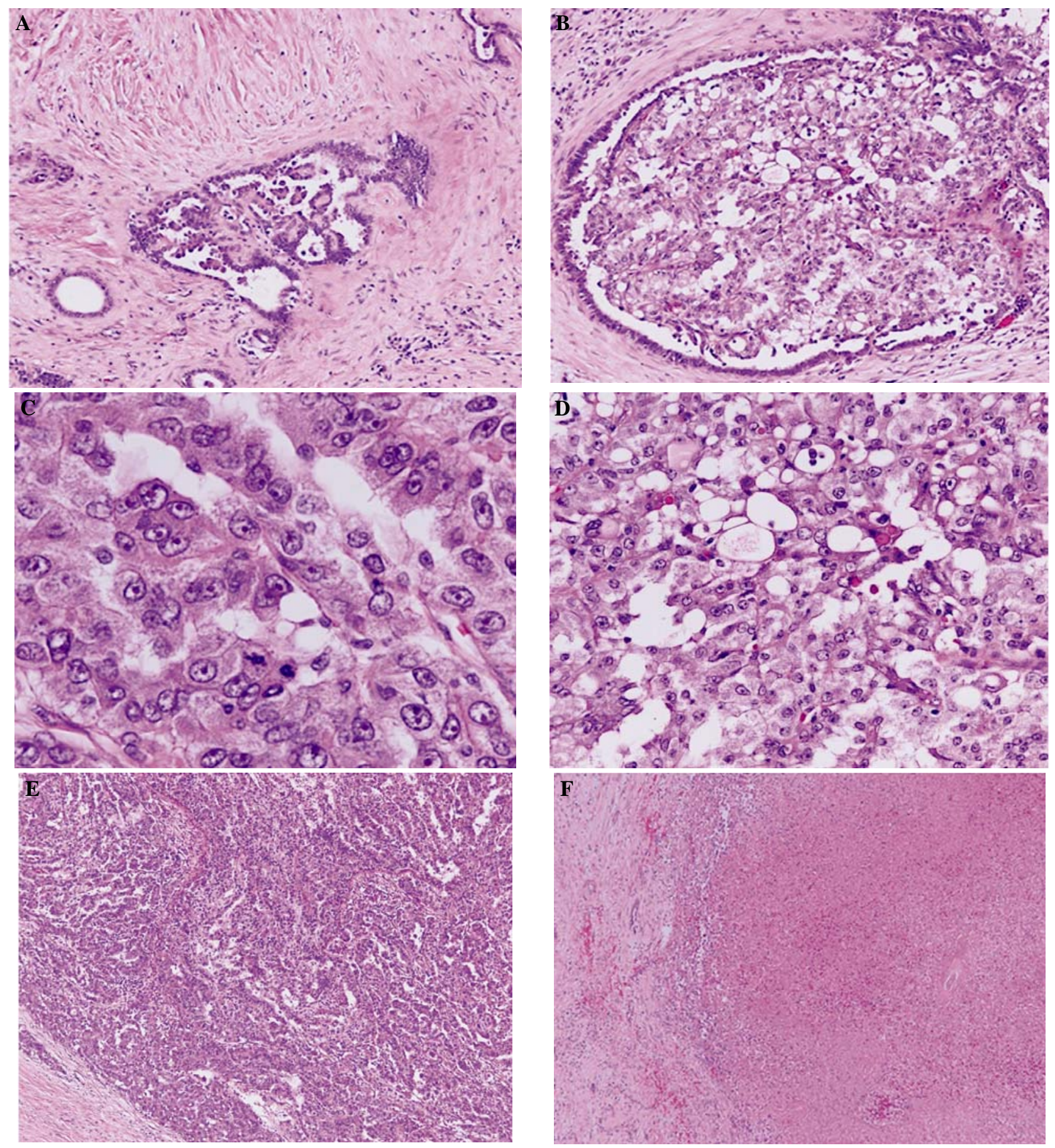

Figure 2. (A-B). The tumor is composed partially of papillary structures surfaced by cuboidal to polygonal cells with clear to eosinophilic cytoplasm. Small glomeruloid structures within cysts are often seen in papillary growth. (C). The cells show marked cellular atypia with conspicuous nucleoli and numerous mitoses. (D). Some areas show a pseudomicrocystic appearance and scattered eosinophilic hyaline globules. (E). In other areas of the tumor, the neoplastic cells are in solid sheets. (F). Areas of extensive necrosis are present. 

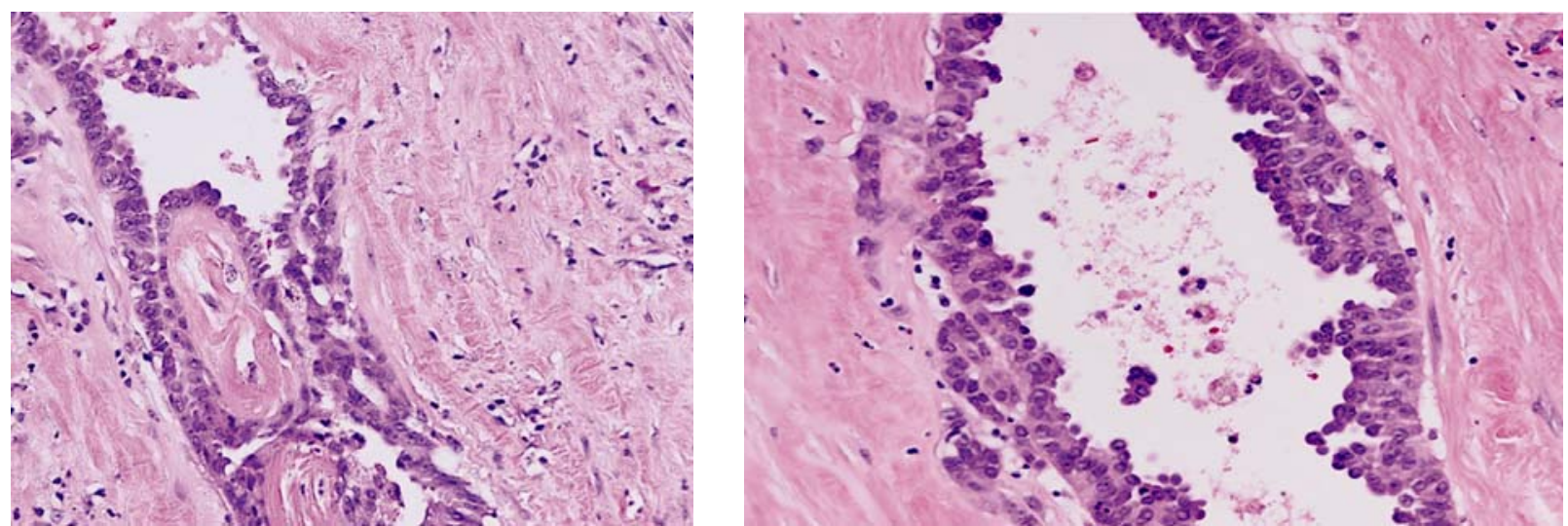

Figure 3. In some foci, adenomatous hyperplasia was evident with tubules lined by several cell layers. H\&E 20×.

Based on the location of the tumor, microscopic morphology, immunohistochemical findings, and past medical history, the diagnosis was primary rete testis adenocarcinoma.
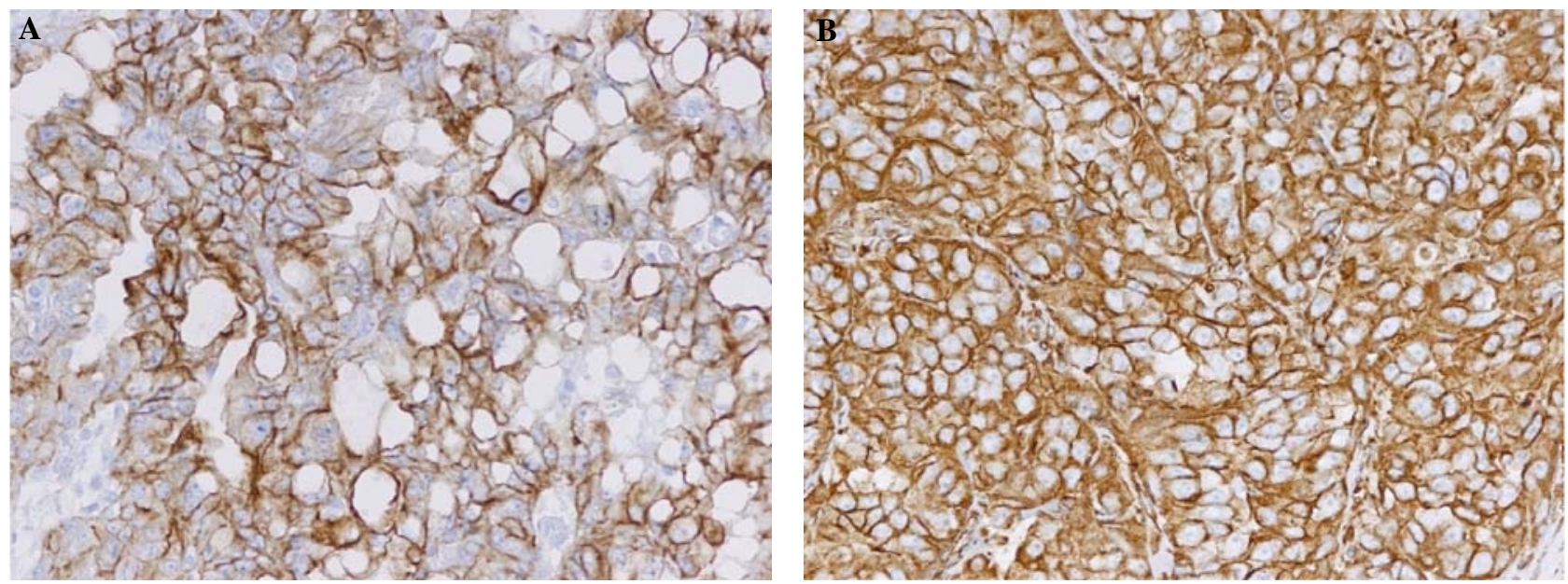

Figure 4. Immunohistochemical staining of the tumor. Cells were positive for (A) Cytokeratin 8, (B) Vimentin and (C) Cytokeratin AE1/AE3.

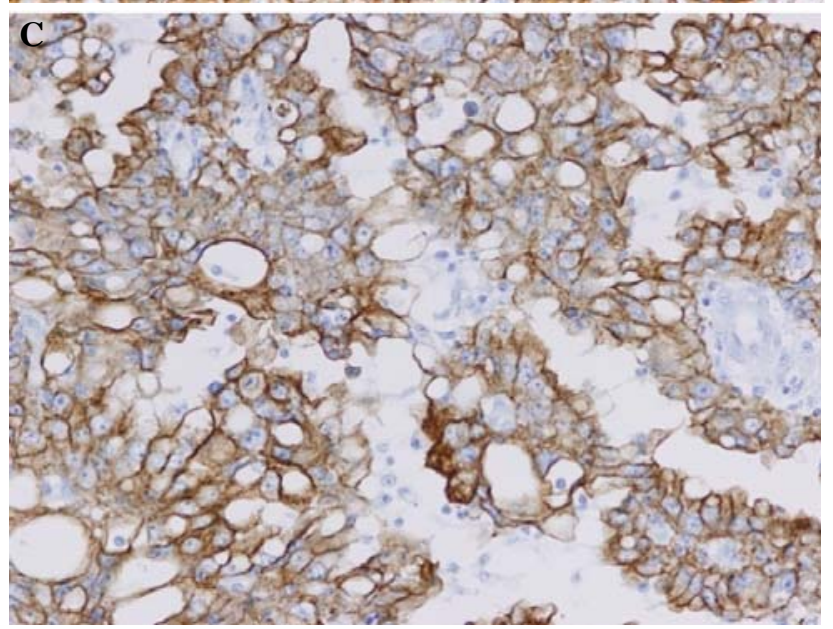

\section{Discussion}

The rete testis is a network of cavernous appearing channels surfaced by flat to low columnar epithelium and located in the mediastinum of the testis. Those channels are surrounded by stroma containing collagen and elastic fibers with occasional myoid and fibroblastic cells ${ }^{[3]}$. 
Among paratesticular malignancies, papillary rete testis adenocarcinoma is rare. Since it is a very rare condition, the medical literature contains only $30-60$ cases ${ }^{[3,4]}$ but not all are certain and some may have represented mesothelioma or papillary serous carcinoma ${ }^{[5]}$. This is an aggressive neoplasm and many patients have metastases at presentation. Metastases are most frequently to lung and bone. The etiology of primary adenocarcinoma of the rete testis is still unclear. An association of adenocarcinoma of the rete testis with exposure to chemical agents such as DEB has been reported only in animal models ${ }^{[6]}$. However, there is a case report which correlates this tumor with long-term exposure to carbon dioxide ${ }^{[7]}$. Adenomatous hyperplasia of the rete testis has been described since $1988^{[8]}$, but our case is the second in which an association between adenomatous hyperplasia of the rete testis and adenocarcinoma was noted ${ }^{[7]}$. This lends support to the proposal that the hyperplastic lesion is a cancer precursor. This tumor has a predilection for older men (age range of 17 to 91 years, with a mean of 54 years), with a scrotal mass being the most common presenting sign ${ }^{[1]}$. Painless scrotal swelling, hydrocele, chronic epididymitis, and inguinal hernia for several years before the diagnosis have been also reported ${ }^{[1]}$. In our case the patient complained of right-sided scrotal swelling for five years, which was followed by a hydrocelectomy and spermatocelectomy.

Nochomovitz and Orenstein propose 5 criteria for rete testis adenocarcinoma ${ }^{[1]}$ : prominent involvement of testicular mediastinum (testicular hilum), 2 transition from normal and hyperplastic epithelium to tumor ${ }^{[3]}$, parietal tunica vaginalis is intact ${ }^{[4]}$, any primary tumor is excluded ${ }^{[5]}$, and predominantly solid gross appearance ${ }^{[2]}$. Recently revised criteria require the immunohistochemical exclusion of malignant mesothelioma or papillary serous carcinoma. Notably, not all rete testis carcinomas are solid; some, like ours, are cystic ${ }^{[5]}$. In large masses, the normal rete epithelium may not show a transition to the tumor.

Microscopically, rete testis adenocarcinoma has been described having various patterns: most commonly tubuloglandular, but retiform (compressed anastomosing tubules), sertoliform (solid tubules), kaposiform (nearly solid with minute thin channels), and biphasic (spindle and epithelioid) patterns also have been described. The presence of glomeruloid structures inside of cystic spaces as in our case (see Figure 1B) has also been noted ${ }^{[5]}$.

The differential diagnosis of rete testis adenocarcinoma morphologically overlaps with metastatic adenocarcinoma, malignant mesothelioma, mullerian-type carcinoma of the testis, and germ cell tumor (see Table). Numerous immunostains showed that the neoplastic cells in our case reacted for pan-cytokeratin, CK8 and vimentin and not for WT-1, cytokeratin 7, and numerous germ cell markers, including OCT3/4, CD30, AFP, PLAP, glypican 3, and CD117. The neoplastic cells showed focal positivity for CD10, LeuM1 and showed questionable weak staining for MOC-31 and BerEP4. A calretinin immunostain was initially negative, but a repeated stain was positive in neoplastic cells. Additional immunostains showed that the tumor was negative for inhibin, CDX-2, TTF-1, p63, PSA and ER.

Mesotheliomas may show architectural similarity to rete testis adenocarcinoma, but can be distinguished based on the location of the lesion and immunohistochemistry profile. Typically malignant mesotheliomas involve the tunica vaginalis, in contrast; adenocarcinoma arises from the mediastinum of the testis. The current case was considered possibly a mesothelioma owing to the positive calretinin staining; however, the positive PAX-8 and LeuM-1 argued against mesothelioma. Mullerian-type carcinomas have been also described in the testis; however, Mullerian stroma was lacking in our case, and the tumor was negative for WT1, CK and ER. The negative germ cell markers in addition to the patient age argue against germ cell tumor.

The differential diagnosis between primary rete testis carcinoma and a metastasis from another primary site may pose a challenge. Metastatic carcinomas would have an interstitial growth pattern, may be multifocal or bilateral, and more often show lymphovascular invasion, all unlike our case. Additionally, specific immunostains for other primary sites, such as CDX-2, TTF-1, PSA and p63 were negative. In our case, the clinical history, in addition to these immunohistochemical results ruled out metastatic adenocarcinoma. Adjacent findings in our case favored a rete testis primary, namely the hyperplastic/preneoplastic changes in the adjacent rete testis (see Figure 3), which appears to be dilated. Also, there are 
reports in the literature of benign rete testis hyperplasia with hyaline globules, papillae and vacuolated cytoplasm, which resemble some of the morphologic changes seen in our tumor. Finally, the tumor is strongly PAX-8 positive, which has been recently described in primary rete testis carcinoma.

In conclusion, given the morphology and immunostaining, our case represents the second primary rete testis adenocarcinoma reported in association with adenomatous hyperplasia of the rete testis.

Table. Use of immunostains to rule in rete testis adenocarcinoma

\begin{tabular}{|c|c|c|c|c|c|c|c|c|c|}
\hline Differential Diagnosis & Pan-CK & Vimentin & AFP & WT-1 & CK7 & CD117 & MOC31 & Calretinin & PAX-8 \\
\hline Adenocarcinoma of Rete Testis & + & + & - & - & - & - & - & + & + \\
\hline Metastatic Adenocarcinoma ${ }^{*}$ & + & & & & & & & & \\
\hline Mesothelioma & - & - & - & + & + & - & + & + & - \\
\hline $\begin{array}{l}\text { Mullerian Type Carcinoma of } \\
\text { the Testis }\end{array}$ & + & - & - & + & - & - & - & - & + \\
\hline Germ Cell Tumor & $+/-$ & - & + & - & - & + & - & - & - \\
\hline
\end{tabular}

Note. ${ }^{*}$ Specific immnunostains for other primary tumors: CDX-2, TTF-1, p63, PSA and ER

\section{Conflict of interest}

The authors declare that they have no competing interests.

\section{References}

[1] Sanchez-Chapado, M., J.C. Angulo, G.P. Haas. Adenocarcinoma of the rete testis. Urology. 1995; 46: 468-75. http://dx.doi.org/10.1016/S0090-4295(99)80257-X

[2] Nochomovitz, L.E., J.M. Orenstein. Adenocarcinoma of the rete testis. Case report, ultrastructural observations, and clinicopathologic correlates. Am J Surg Pathol. 1984; 8: 625-34. PMid: 6465420. http://dx.doi.org/10.1097/00000478-198408000-00006

[3] Wu CA, Chen YH, Man, KM, Shen JL, Chen WC. Papillary adenocarcinoma of rete testis mimics inflammatory lump. Case Rep Urol. 2011: 857812. PMid: 22606627.

[4] Hagiuda, J., et al. Adenocarcinoma of the rete testis. A case report. Nihon Hinyokika Gakkai Zasshi. 2010; 101: 749-53. PMid: 21174741. http://dx.doi.org/10.5980/jpnjurol.101.749

[5] Amin, M.B. Selected other problematic testicular and paratesticular lesions: rete testis neoplasms and pseudotumors, mesothelial lesions and secondary tumors. Mod Pathol. 2005; 18 Supp1 2: S131-45. PMid: 15502808. http://dx.doi.org/10.1038/modpathol.3800314

[6] Newbold, R.R., B.C. Bullock, J.A. McLachlan. Adenocarcinoma of the rete testis. Diethylstilbestrol-induced lesions of the mouse rete testis. Am J Pathol. 1986; 125: 625-8. PMid: 3799821.

[7] Gruber, H., et al. Adenocarcinoma of the rete testis: report of a case with surgical history of adenomatous hyperplasia of the rete testis. J Urol. 1997; 158:1525-6. http://dx.doi.org/10.1016/S0022-5347(01)64262-9

[8] Nistal, M., R. Paniagua. Adenomatous hyperplasia of the rete testis. J Pathol. 1988; 154: 343-6. PMid: 3385514. http://dx.doi.org/10.1002/path.1711540410 